\title{
Nuclear Electromagnetic Generator: Introduction in Charge Algebra and Elements of Charge Kinetics
}

\author{
V. Yu. Tertychny-Dauri \\ Department of Physics and Engineering, Saint-Petersburg National Research University of Information Technologies, Mechanics \\ and Optics, Saint-Petersburg, Russia \\ Email: tertychny-dauri@mail.ru
}

How to cite this paper: Tertychny-Dauri, V.Yu. (2018) Nuclear Electromagnetic Generator: Introduction in Charge Algebra and Elements of Charge Kinetics. Open Access Library Journal, 5: e4614. https://doi.org/10.4236/oalib.1104614

Received: April 21, 2018

Accepted: May 14, 2018

Published: May 17, 2018

Copyright (c) 2018 by author and Open Access Library Inc.

This work is licensed under the Creative Commons Attribution International License (CC BY 4.0).

http://creativecommons.org/licenses/by/4.0/

\section{cc) (†) Open Access}

\begin{abstract}
This work is devoted to solution of some problems connected with kinetic equations of reproduction for charged particles and charged splinters of the fission inside of nuclear toroidal electromagnetic generator (nuclegen). There are discussed questions of solutions behaviour for charged equations. Special attention is paid to charge kinetics of the nuclegen under the influence of small random disturbances and stimulated by this influence to analysis of stochastic motion properties for the ionite gas.
\end{abstract}

\section{Subject Areas}

Theoretical Physics, Modern Physics, Nuclear Physics

\section{Keywords}

Charge Kinetics, Kinetics of Charged Particles and Charged Splinters of the Nuclear Fission

\section{Introduction}

We consider a series of questions connected with composition and solution of kinetic equations for chain nuclear fission products into active zone of nuclear toroidal electric generator [1] [2] [3]. Of course, by spelling of kinetic equations for the reproduction of charged particles and charged splinters of fission this consideration would be accompanied by great share of the convention. The main aim, which here is placed, consists in the description of general regularity of reproduction process and not at all the counting of exact charges quantity up to last electron and proton. 
It is important to note, in our view, that on dynamics of growth of charged particles $Q$ number the governing action the growth of number prompt and delayed neutrons $N$ into active zone toroid will be exercising so far as the each new fission act by neutrons will be leading to the new fission of nucleuses and to the formation of another charged particles and charged splinters.

In Sections 2-4, the conclusion of charged kinetic equations for electric generator is kept. Besides the finding equations there are established exponential instability of charged equations solutions for the coefficient of ionites reproduction which over one.

Investigation of charged kinetic equations is going on in Sections 5-9 also when the initial kinetic system is criticized by the influence of small random disturbances. Analysis of stochastic motion properties succeeded come to known scheme with action functional and to finding of corresponding kinetic quasipotential. Initial problem hasn't analytical solutions in the class of free stable kinetic matrixes. Therefore there is proposed replacement method of the initial problem on the similar problem of kinetics with stable normal matrix.

\section{Charge Kinetics of a Nuclear Electric Generator}

Scaled demands at wide application of electric energy dictate the necessity to produce nuclear energy by means of fast-neutron electric generator. These nuclear devices are the most favourable due to their high values of the reproduction coefficient.

However, the use of fast-neutron electric generators challenges as there are several demands to maintain the nuclear kinetic process at a fitting level. Above all, it is necessary to keep high average energy of neutrons at the level of several hundred kilovolts [4] [5]. Here the energy spectrum of neutrons is defined by the properties of combustible fuel, raw materials and construction materials in relation to the inelastic scattering of the neutrons $\left({ }^{238} \mathrm{U}\right.$ has a big cross-section of inelastic scattering). In addition, to achieve criticality the operation of fast-neutron nuclear devices needs a high concentration of a combustible fuel in relatively small volumes. The latter is caused by low values of the fission cross-section in the area of high energies.

Therefore, our further reasoning is based on the admission that the nuclear device (nuclear electric generator or nuclegen) has a breeder basis.

For simplicity and convenience all charged splinters and particles of both signs (ions, electrons, protons etc.) are called ionites, and their aggregate in quantity $Q$ is ionite gas (ionite gas cord).

As in the case of neutrons, the balance of ionites is characterise by the ionite reproduction coefficient $f$. The reproduction coefficient $f$ is defined as the ratio of the number of ionites in any generation to the number of ionites in the previous generation.

The average lifetime of one generation of ionites $r$ is supposed to be proportional to the average lifetime of one generation of neutrons $l$ : 


$$
r=\lambda l+F(\varepsilon, v, \delta, \mu)
$$

in which $\lambda>0$ is a proportionality coefficient, $(\varepsilon, v, \delta, \mu)$ is a set of parameters characterizing the electromagnetic influence, air possible residual drag of the medium, dynamic and construction features respectively, all influencing the value $r$.

For all intents and purposes the dependence (1) may possess not a constant but a rather complex function form with all values included being dependent on time and spatial variables in some way. In work [1] to analize the simplest charge kinetics value $\lambda=1$ is fixed so that value $r$ coincides with value $l$ and is entirely defined by it. Apparently, this does nothold true what is evidenced by the following the correlation (1).

Unlike the neutron losses (capture without fission, leakage etc.), the losses of ionites, that control their lifetime, are determined by their neutralization degree (i. e. missed charges capture). The neutralization degree depends on the general field $(H, E)$, particles velocity etc.

If $f=1$, nuclear fission with the formation of ionites and neutrons occurs steadily at constant reaction rate $(k=1, k$ is the coefficient of neutrons reproduction); its active zone is critical with a self-sustained chain reaction. This case implies $Q=$ const, elements of inner electromagnetic field $(H, E)$ including $I_{*}, j_{*}$ are also close to constant values.

Ignoring delayed ionites the density change of ionite gas $q$ in active zone within one generation equals to $f q-q=(f-1) q$. If $r$ is an average life time of one ionite generation, it means that per time unit the density of charged lively particles $q$ changes in $(f-1) / r$ times, i.e.

$$
\frac{\mathrm{d} q}{\mathrm{~d} t} \frac{(f-1) q}{r},
$$

where $q(t)=q_{0} \exp \left(t / T_{*}\right)$, with $q_{0}$ is initial ionite density, $T_{*}=r /(f-1)$ is charge period of nuclear electric generator.

\section{Linear Theory of Charge Kinetics}

As mentioned above delayed neutrons play a crucial role and have a practical application in the controlled process of nuclear chain reaction. The fact that delayed neutrons alon with other $\beta$ descendants contribute into ionite gas formation is obvious.

Let $\gamma$ is delayed ionite part (charged $\beta^{-}$descendants), $(1-\gamma)$ is instant ionite part. The reproduction coefficient $f$ can be noted as a coefficient consisting of the two parts: $f=f_{0}+f_{*}$, where $f_{0}=(1-\gamma) f, f_{*}=\gamma f$. After $\beta$-disintegration decay of splinters the delayed ionites become instant. this means that their effective lifetime $r_{*}$ equals the sum: $r_{*}=r_{\beta}+r_{0}$, where $r_{\beta}$ is delay time ( $\beta$-disintegration time), $r_{0}$ is instant ionite life time.

Hence, average effective lifetime of $r$ ionite generation equals

$$
r=\gamma r_{*}+(1-\gamma) r_{0} \approx r_{0}+\gamma r_{*} .
$$


Since $\gamma r_{*} \gg r_{0}$, the charge period of nuclear electric generator $T_{*}=r /(f-1)$ is determined by the average time of ionite delay.

The simplest linear theory of charge kinetics needs more thorough consideration. The density of ionite gas $q$ is admitted to be the blend of densities of charged particles and fission splinters of the two types mentioned above, i.e. $q=q_{m}+q_{z}$. Here $q_{m}$ is instant ionite density, $q_{z}$ is delayed ionite density. Then instead of the correlation (2) ignoring the presence of delayed ionites the system of two kinetic equations is possible to be written. These equations describe how the dynamics of ionite gas density (concentration) changes in an nuclear electric generator:

$$
\begin{gathered}
\frac{\mathrm{d} q_{m}}{\mathrm{~d} t}=\frac{[(1-\gamma) f-1] q_{m}}{r}+\frac{q_{z}}{r_{*}}, \\
\frac{\mathrm{d} q_{z}}{\mathrm{~d} t}=\frac{\gamma f q_{m}}{r}-\frac{q_{z}}{r_{*}}
\end{gathered}
$$

with initial conditions

$$
\begin{aligned}
& \left.q_{m}\right|_{t=0}=q_{m o},\left.\quad \frac{\mathrm{d} q_{m}}{\mathrm{~d} t}\right|_{t=0}=q_{m o}^{\prime}, \\
& \left.q_{z}\right|_{t=0}=q_{z 0},\left.\quad \frac{\mathrm{d} q_{z}}{\mathrm{~d} t}\right|_{t=0}=q_{z 0}^{\prime} .
\end{aligned}
$$

The right parts of Equations (3) and (4) should be commented. In equation (3) coefficient $q_{m}$ characterizes the density change of instant ionite number in lifetime of one generation. The second summand characterizes the growth of value $q_{m}$ at the cost of $q_{z}$ by the transition of the latter into the category of instant ionites. Equation (4) describes the density change of delayed ionites. In this equation the first summand shows the increase of the concentration of delayed ionites at the cost of formation instant ionites; the second one, on the contrary, shows the decrease of the concentration of the amount of delayed ionites within their lifetime (i.e. within the time of $\beta^{-}$-decay). As well seen in the system (3), (4) the density (the concentration of the amount) of delayed ionites $q_{z}$ creates the feedback in relation to the density of instant ionites $q_{m}$.

Following some simple transformations, the system of Equations (3), (4) is reduced to a homogenous differential equation of the second order with constant coefficients. The equation describes how to regulate kinetics peculiarities of a nuclear electric generator:

$$
\ddot{x}+\delta_{1} \dot{x}-\delta_{2} x=0
$$

in which $x=q_{m}$ (or $\left.x=q_{z}\right)$,

$$
\delta_{1}=\frac{r+r_{*}-(1-\gamma) f r_{*}}{r r_{*}}, \quad \delta_{2}=\frac{f-1}{r r_{*}} .
$$

The characteristic equation has two real roots of a clearly determined sign $(f \geq 1)$ : 


$$
\begin{aligned}
& \lambda_{1}=\frac{-\delta_{1}+\left(\delta_{1}^{2}+4 \delta_{2}\right)^{1 / 2}}{2} \geq 0, \\
& \lambda_{2}=\frac{-\delta_{1}-\left(\delta_{1}^{2}+4 \delta_{2}\right)^{1 / 2}}{2}<0 .
\end{aligned}
$$

Hence, considering the starting conditions (5) the kinetics equations have the following solutions as functions of time:

$$
\begin{aligned}
& q_{m}(t)=\left(\frac{q_{m 0}^{\prime}-\lambda_{2} q_{m 0}}{\lambda_{1}-\lambda_{2}}\right) \mathrm{e}^{\lambda_{1} t}+\left(\frac{\lambda_{1} q_{m 0}-q_{m 0}^{\prime}}{\lambda_{1}-\lambda_{2}}\right) \mathrm{e}^{\lambda_{2} t}, \\
& q_{z}(t)=\left(\frac{q_{z 0}^{\prime}-\lambda_{2} q_{z 0}}{\lambda_{1}-\lambda_{2}}\right) \mathrm{e}^{\lambda_{1} t}+\left(\frac{\lambda_{1} q_{z 0}-q_{z 0}^{\prime}}{\lambda_{1}-\lambda_{2}}\right) \mathrm{e}^{\lambda_{2} t} .
\end{aligned}
$$

In solutions (6) for $q_{m}$ and $q_{z}$ the second summand in the right part quicly approaches to zero. Disregarding it, growth dynamics of density (of the number) of ionites $q_{m}$ and $q_{z}$ is supposedly defined by the first summand in the solutions (6). As mentioned above, delayed ionites slow the transient process significantly, and the charge period $T_{*}=r /(f-1)$ is almost entirely defined by the average time of delay $r_{*}$. This feature is also notable in the correlations (6). Indeed, the following approximations

$$
r_{*} \sim r, \quad \delta_{1} \sim \frac{2-f}{r}, \quad \delta_{2} \sim \frac{f-1}{r^{2}},
$$

prove the evaluations

$$
q_{m}(t), q_{z}(t) \sim \mathrm{e}^{\lambda_{1} t} \sim \mathrm{e}^{t / T_{*}} .
$$

\section{Some Preliminary Results}

Thus, a rough kinetics analysis of nuclear chain fission in vacuum toroidal shaped electric generator has shown that the achievement of exponential density growth (number) of charged particles moving at high rate and charged splinters may be considered proven. The boost of ionite gas density results in hasty growth of induced of electrical current in the external winding of a nuclear electric generator. It happens according to the electrodynamics peculiarities mentioned above (see the author's article [3], correlations (17)).

In agreement with the principals nuclear electrodynamics the solutions to modified Maxwell's equations (the work [3], formulae (7), (8)) for a general electric field can be found in the form

$$
H=H_{m}(x, y, z) \mathrm{e}^{a_{m} t}, \quad E=E_{m}(x, y, z) \mathrm{e}^{b_{m} t},
$$

where $a_{m}, b_{m}$-constant positive coefficients, $m=\overline{1, \infty}$. If the Fourier transform of the distribution of field is known at the initial moment of time

$$
H^{0}(x, y, z)=\sum_{m} c_{m} H_{m}(x, y, z), \quad E^{0}(x, y, z)=\sum_{m} d_{m} E_{m}(x, y, z)
$$

along with the full system of mutually orthogonal vector functions $H_{m}, E_{m}$, then a general solution to the problem of unlimited growth of electromagnetic field 
can be presented using Equation (7) in the type of the following divergent series:

$$
\begin{aligned}
& H(x, y, z, t)=\sum_{m} c_{m} \mathrm{e}^{a_{m} t} H_{m}(x, y, z), \\
& E(x, y, z, t)=\sum_{m} d_{m} \mathrm{e}^{b_{m} t} E_{m}(x, y, z) .
\end{aligned}
$$

Currently, the possibilities of nuclear power engineering are restricted by chemical and thermal transformations at a significant low level. At the same time physics of nuclear electric engineering today is boosting the amount of neutrons fission in a reactor core as it inevitably results in overheating and thermal explosion.

This paper describes an alternative scheme for vacuum conducting of a chain nuclear reaction in quickly increasing and directional electromagnetic field. Under considered conditions there is nothing to heat except for cylindrical walls of a toroid. However, this problem is solved due to the presence of directional electromagnetic field that screens cascade currents of neutrons and ionite gas inside a nuclear generator.

\section{Charge Kinetics under the Action of Small Random Disturbances}

Here the problem of stochastic stability under the action of small random disturbances of initial kinetic process is going to be considered [6] [7] [8] [9]. Stochastic fluctuations in a dynamic system behavior (3), (4) may vary by nature. They may result from the deformation of outer and inner electromagnetic fields, changes of thermal and vacuum regimes, chemical content of active nuclear components of kinetic process, constructive imperfections or operation inertance of control devices. The diversity of reasons causing slight, negligible disturbances to appear can lead to one general catastrophic overall result implying the destruction of the whole kinetic system itself.

The investigation of stochastic stability (instability) of the systems (3), (4) regarding small random disturbances needs revising some basic concepts.

\section{Asymptotic Stability and Stochastic Instability}

Unperturbed deterministic kinetic system (3), (4) is considered here:

$$
\dot{x}=A x, \quad x(0)=x_{0},
$$

where

$$
\begin{gathered}
A=\left(\begin{array}{ll}
a_{11} & a_{12} \\
a_{21} & a_{22}
\end{array}\right), \quad x=\left(\begin{array}{c}
q_{m} \\
q_{z}
\end{array}\right), \\
a_{11}=\frac{(1-\gamma) f-1}{r}, \quad a_{21}=\frac{\gamma f}{r}, \quad a_{12}=-a_{22}=\frac{1}{r_{*}},
\end{gathered}
$$

which at the origin of coordinates (at zero point identifiable with a two-dimensional zero) has the stability position: $0=A 0$.

The stability position (zero point) is stable (Lyapunov stable) if for any 
neighborhood $W: 0 \in W$ there is a neighborhood $W_{0} ; x_{0} \in W_{0}$ for which equation solutions are (8) $x(t) \in W, t \geq 0$. If, moreover, $x(t) \rightarrow 0$ at $t \rightarrow \infty$, then the stability position is called asymptotically stable.

Then it is assumed that the system (8) is perturbed by small white noise

$$
\dot{X}=A X+\varepsilon \dot{w}, \quad X(0)=x_{0},
$$

where $w(t)$ is a two-dimensional Wiener process, $\varepsilon>0$ is a small numerical parameter. The problem of trajectories exit of process $X(t)$ from limited region $D$ with stability position is of great interest. The appearance of a small parameter in system (9) can by explained with an assumption that the noise intensity is low compared to deterministic factors affecting the system dynamics.

If matrix $A$ is stable (Hurwitz matrix), i.e. its own numbers $\lambda_{1}$ and $\lambda_{2}$ have negative real parts. For this condition it is necessary and satisfactory [10] that coefficients $\delta_{1}, \delta_{2}$ of characteristical equation $\lambda^{2}+\delta_{1} \lambda-\delta_{2}=0$ fulfiled the following inequalities

$$
\delta_{1}=\frac{r+r_{*}-(1-\gamma) f r_{*}}{r r_{*}}>0, \quad \delta_{2}=\frac{f-1}{r r_{*}}<0 .
$$

It is obvious that the inequalities are held if $f<1$. As matrix $A$ is considered as stable, the stability position of the unperturbed system (origin of coordinates) is asymptotically stable:

$$
|x(t)|=\left|\mathrm{e}^{A t} \cdot x_{0}\right| \leq\left|\mathrm{e}^{A t}\right| \cdot\left|x_{0}\right| \rightarrow 0 \text { when } t \rightarrow \infty .
$$

It is worth mentioning that vector function $\dot{w}(t), t \geq 0$ is not norm limited. Consequently solutions $X(t)$ to Equation (9) may leave from any neighborhood of the stability position. Even with the presence of asymptotic stability of this stability position [11], i.e. trajectories $X(t)$ with probability 1 may deviate too far from the stability position. Thus, the system becomes unstable. To make the term "instability" meaningful some concepts are to be introduced using definitions and research schemes from [11].

Denote the region $D \subset \mathbf{R}^{2}$ is region if state vector $X(t) \in D$, then object (9) functions; if the phase vector leaves $D$, then the object destroys. The region $D$ is called critical region.

Introduce random value $\tau=\min \{t: X(t) \notin D\}$ is the time before system (9) destruction, where $X(t)$ is its solution with initial condition $x_{0}$ or in other way $\tau$ is the first moment of process exit from $D$. Probability value $P\{\tau \leq T\}$ can be taken as an instability measure, if time interval $[0, T]$ of the object functional ability is fixed. Otherwise, mathematical expectation value of $E \tau$ can be taken as an instability measure, if the time interval is unknown. The estimations of $P\{\tau \leq T\}, E \tau$ are given with the aid of action functional.

\section{Action Functional and Quasipotential}

Denote by $C_{0 T}\left(\mathbf{R}^{2}\right)$ a set of continuous functions within time interval $[0, T]$ with values in $\mathbf{R}^{2}$. In this space the metric $\rho_{0 T}(\varphi, \psi)=\sup _{0 \leq t \leq T}\|\varphi(t)-\psi(t)\|$ should be considered and functional for absolutely continuous functions 
$\varphi(t) \in C_{0 T}\left(\mathbf{R}^{2}\right)$ should be defined

$$
S_{0 T}(\varphi)=\frac{1}{2} \int_{0}^{T}\|\dot{\varphi}(s)-b(\varphi(s))\|^{2} \mathrm{~d} s, \quad b(\varphi) \in \mathbf{R}^{2},
$$

If function $\varphi(t)$ is not absolutely continuous within $[0, T]$ or the integral (10) is divergent, then we suppose $S_{0 T}(\varphi)=+\infty$.

For example, in the case of classical mechanical system for which movement along trajectory $\varphi(t)$ is a functional $S(\varphi)$ motion of this system occurs along the extremals of functional $S(\varphi)$. For a unperturbed dynamic system have

$$
\dot{\varphi}=b(\varphi), \quad \varphi(0)=\varphi_{0}, \quad b(\varphi) \in \mathbf{R}^{2},
$$

it means that if $S_{0 T}(\varphi)=0$, then function $\varphi(t)$ in $[0, T]$ is the trajectory of system (11) as $\varphi(t)$ is absolutely continuous within $[0, T]$ and satisfies almost everywhere Equation (11).

Let the $D$ is a limited in $\mathbf{R}^{2}$ (critical) region and $\partial D$ is its smooth boundary. If trajectory $\varphi(t)$ of system (11) starting at point $\varphi_{0} \in D$ leaves $D \cup \partial D$ in a finite time, trajectories of process $\Phi(t)$ :

$$
\dot{\Phi}=b(\Phi)+\varepsilon \dot{w}, \quad \Phi(0)=\varphi_{0}, \quad \varepsilon>0,
$$

coming from $\varphi_{0}$ also with probability close to 1 with small $\varepsilon$ leave region $D$ within this time. The first exit is more likely to occur near the exit point from $D$ trajectory $\varphi(t)$ of system (11).

More precise definition of this result is connected to the approximation that happens while calculating probabilities of events $\{\|\Phi(t)-\varphi(t)\|<\delta\}$ :

$$
P\{\|\Phi(t)-\varphi(t)\|<\delta\} \approx \mathrm{e}^{S_{0 T}(\varphi) / \varepsilon^{2}}
$$

with small $\varepsilon, \delta>0$ for functional $S_{0 T}(\varphi)$ (10) for time interval $[0, T]$. Functional $S_{0 T}(\varphi) / \varepsilon^{2}$ with constant norming $\varepsilon^{2}$ is stated as action functional for a set of random processes $\Phi(t)$ by analogy with quantum mechanics problems [12] and described with stochastic Equation (12).

If point $0 \in \mathbf{R}^{2}$ is an asymptotically stable stability position of system (11), the quasipotential of system (11) relative to point 0 is function $V\left(0, \varphi_{0}\right)$ determined by equality

$$
V\left(0, \varphi_{0}\right)=\inf S_{0 T}(\varphi),
$$

The functional of action is given by Equality (10) with

$$
\varphi \in C_{0 T}\left(\mathbf{R}^{2}\right),\left.\varphi\right|_{t=0}=0,\left.\quad \varphi\right|_{t=T}=\varphi_{0},
$$

and the ends of the interval $[0, T]$ are not fixed. Have: function $V\left(0, \varphi_{0}\right) \geq 0, V(0,0)=0$ and $V\left(0, \varphi_{0}\right)$ is continuous.

The essential property of the quasipotential is given the statement concluding the following. If vector field $b(\varphi)$ in system (11) allows for decomposition

$$
b(\varphi)=-\nabla U(\varphi)+v(\varphi)
$$


with gradient operator $\nabla$. Here potential function $U(\varphi)$ is continuously differential in $D \cup \partial D, U(0)=0, U(\varphi)>0, \nabla U(\varphi) \neq 0$ when $\varphi \neq 0,(\nabla U(\varphi), v(\varphi))=0$. This means:

1) quasipotential $V\left(0, \varphi_{0}\right)$ equals to

$$
V\left(0, \varphi_{0}\right)=2 U\left(\varphi_{0}\right)
$$

for all $\varphi_{0} \in D \bigcup \partial D$ so that $U\left(\varphi_{0}\right) \leq U_{0}=\min _{y \in \partial D} U(y)$;

2) the only extremal $\hat{\varphi}(t)$ of functional $S_{0 T}(\varphi)$ at a set of functions $\varphi(t)$ is defined by equation

$$
\dot{\varphi}=\nabla U(\varphi)+v(\varphi)
$$

where $\left.\varphi\right|_{t=0}=0,\left.\varphi\right|_{t=T}=\varphi_{0}, t \in[0, T]$.

It can be shown that for any curve $\varphi(t)$ connecting points 0 and $\varphi_{0}$ the following un equation holds

$$
S_{0 T}(\varphi) \geq 2 U\left(\varphi_{0}\right)=S_{0 T}(\hat{\varphi}),
$$

i.e. $V\left(0, \varphi_{0}\right)=\inf S_{0 T}(\varphi)=2 U\left(\varphi_{0}\right)$ and function $\hat{\varphi}(t)$ as the only solution of Equation (15) is the only extremal.

In addition, it is possible to show that asymptotics of average time of process exit $\Phi(t)$ (12) from critical region $D$ is determined by limiting equality

$$
\lim _{\varepsilon \rightarrow 0} \varepsilon^{2} \ln E \tau=V_{0}=\min _{y \in \partial D} V(0, y),
$$

where $V(0, y)$ is found quasipotential of dynamic system (11) relative to stability position (point 0 ) which is assumed as asymptotically stable.

\section{Kinetic Quasipotential}

The initial kinetic system (8) with a known stable constant matrix of coefficients $A$ is worth mentioning again. Let the vector field $b(x)=A x$ permits the decomposition of type (14):

$$
A x=-\nabla(B x, x)+C x,
$$

in which $U(x)=(B x, x), v(x)=C x ; B, C$ are non-zero square matrices of dimension 2 to defined. The matrix $B$ is assumed to be a positive definite matrix. Considering the results of the previous section, there appears a task to find matrices $B$ and $C$, potential $U(x)$, quasipotential $V\left(0, x_{0}\right)$ and extremal equation of the type (15).

From the correlation (16) there is an equation

$$
A=-2 B+C,
$$

and from the condition of orthogonality $(\nabla U(x), v(x))=0, \forall x \in \mathbf{R}^{2}$, there is obtained

$$
C^{*} B=0,
$$

in which $C^{*}$ is a matrix transposed in relation to $C$.

The equations system (17), (18) leads to a square matrix equation in $B: A^{*} B+2 B^{*} B=0$, which has not analytical solution for an arbitrary value of 
matrix $A$ (i.e. a solution that does not lead to $C=0$ ). It is worth mentioning it is possible to arrive to the same equation from the Jacobi equation for a quasipotential under the condition of orthogonality of vectors $\nabla U(x)$ and $v(x)$, in which $v(x)=A x+\nabla U(x), \quad V(0, x)=2 U(x)$ :

$$
\frac{1}{2}\|\nabla V(0, x)\|^{2}+(A x, \nabla V(0, x))=0 .
$$

If $A$ is a normal matrix (that is, $A A^{*}=A^{*} A$ ) with a negative definite symmetrical matrix $A+A^{*}$, a solution can be found and take the form

$$
\begin{gathered}
B=-\frac{1}{4}\left(A+A^{*}\right), \quad C=\frac{1}{2}\left(A-A^{*}\right), \\
U(x)=-\frac{1}{4}\left(\left(A+A^{*}\right) x, x\right), \quad V\left(0, x_{0}\right)=2 U\left(x_{0}\right) .
\end{gathered}
$$

The extremal equation also belongs to the same case when the $A$ being a normal matrix:

$$
\dot{\varphi}=\nabla U(\varphi)+v(\varphi)=-A^{*} \varphi
$$

To ensure the condition of orthogonality it is necessary to place a enough strict restriction upon matrix $A$ as its normality condition:

$$
\begin{aligned}
& \left(-\frac{1}{2}\left(A+A^{*}\right) x, \frac{1}{2}\left(A-A^{*}\right) x\right) \\
& =-\frac{1}{4}\left[(A x, A x)-\left(A^{*} x, A^{*} x\right)\right]=-\frac{1}{4}\left[\left(A^{*} A x, x\right)-\left(A A^{*} x, x\right)\right]=0 .
\end{aligned}
$$

The matrix $A$ is not normal in the initial task. Consequently, we arrive to the situation which has a numerical solution in the case to be considered. A more optimistic prognosis is connected to an approximation task, a theoretical analogue of an approximate numerical method in a sense. It deals with a substitution of initial system (8) for a kinetic model which is asymptotically close to it.

\section{Approximative Kinetic System}

At first, matrix $A$ cannot be a normal one even under the condition with the reproduction coefficient of ionites $f<1$. As said above, in this case matrix $A$ is stable. Normality of $A$ means its symmetry, i.e. the resulting in the equality of elements $a_{12}=a_{21}$ :

$$
\frac{\gamma f}{r}=\frac{1}{r_{*}}
$$

from which

$$
f=\frac{r}{\gamma r_{*}}=\frac{\gamma r_{*}+(1-\gamma) r_{0}}{\gamma r_{*}}=1+\Delta,
$$

in which it is designated as $\Delta=(1-\gamma) r_{0} /\left(\gamma r_{*}\right)$.

An important thing is that value $\Delta$ can be given the "status" of a small parameter. Indeed, the previous appraisements imply that 


$$
0<\Delta=\frac{(1-\gamma) r_{0}}{\gamma r_{*}} \approx \frac{r_{0}}{\gamma r_{*}} \ll 1 .
$$

Therefore, matrix $A$ is to be approximated by a stable negative definite normal matrix $\bar{A}$ :

$$
A=\bar{A}+A_{\delta}, \quad A_{\delta}=\left(\begin{array}{ll}
0 & \delta \\
0 & 0
\end{array}\right),
$$

where $\delta>0$ is a small parameter.

The choice of algorithm of approach (19) is to be the following. Let there be coefficient $f<1$, more precisely $f=1-\Delta$ in a normal matrix $\bar{A}$ :

$$
\bar{A}=\bar{A}^{*}=\left(\begin{array}{cc}
\frac{(1-\gamma) f-1}{r} & a \\
\frac{\gamma f}{r} & -\frac{1}{r_{*}}
\end{array}\right), \quad A=\left(\begin{array}{cc}
\frac{(1-\gamma) f-1}{r} & \frac{1}{r_{*}} \\
\frac{\gamma f}{r} & -\frac{1}{r_{*}}
\end{array}\right),
$$

where

$$
\frac{\gamma f}{r}=a, \quad a+\delta=\frac{1}{r_{*}} .
$$

The obtained equations are the following

$$
\begin{gathered}
a=\frac{\gamma(1-\Delta)}{r}=\frac{\gamma-(1-\gamma) r_{0} / r_{*}}{\gamma r_{*}+(1-\gamma) r_{0}}=\frac{1}{r_{*}} \cdot \frac{1-\Delta}{1+\Delta}, \\
\delta=\frac{1}{r_{*}}-a=\frac{1}{r_{*}}-\frac{1}{r_{*}} \cdot \frac{1-\Delta}{1+\Delta}=\frac{1}{r_{*}} \cdot \frac{2 \Delta}{1+\Delta} .
\end{gathered}
$$

It is obvious that $a \sim 1 / r_{*}, \delta>0$ is a small parameter because $\Delta>0$ is a small parameter here.

Thus, initial kinetic system (8) with a "small" disturbing vector $A_{\delta} x,\left\|A_{\delta} x\right\| \rightarrow 0$ for $t \rightarrow \infty$ (in a limited time interval $\left\|A_{\delta} x\right\| \rightarrow 0$ is provided for $\delta \rightarrow 0$ ) of the type

$$
\dot{x}=\bar{A} x+A_{\delta} x, \quad x(0)=x_{0},
$$

is given approximation by a "normal" stable kinetic system

$$
\dot{x}=\bar{A} x, \quad x(0)=x_{0} .
$$

Further, the scheme described above starts to operate for studying stochastic motion of system (20) disturbed with small white noise

$$
\dot{X}=\bar{A} X+\varepsilon \dot{w}, \quad X(0)=x_{0}
$$

with the aid of quasipotential theory. For system (20) quasipotential relatively to stability position $x=0$ has the type

$$
V(0, x)=-\left(\left(\bar{A}+\bar{A}^{*}\right) x, x\right)=-(\bar{A} x, x),
$$

where $\bar{A}$ is stable negatively defined normal (symmetrical) matrix. The equation for extremals has the form

$$
\dot{\varphi}=-\frac{1}{2}\left(\bar{A}+\bar{A}^{*}\right) \varphi+\frac{1}{2}\left(\bar{A}-\bar{A}^{*}\right) \varphi=-\bar{A}^{*} \varphi=-\bar{A} \varphi .
$$


This allows to draw the following conclusion. Markov trajectories $X(t)$ of system (21) with the probability tending to 1 for $\varepsilon \rightarrow 0$ first leave critical region $D$ near of point $y_{0} \in \partial D$, i.e. near of the extremal starting at this point: $\hat{\varphi}(t)=\exp (-\bar{A} t) \cdot y_{0}$.

\section{Conclusion}

The moment to sum up has come. The main and principal conclusion is contained in following: in this work, the inference of kinetic equations describing the reproduction process for charged particles and charged splinters inside of nuclear electromagnetic generator is conducted. Moreover, the description of solutions behaviour of charged equations in the form of ionite gas motion is realized. Exact analytical questions of the stable stochastic motion of ionite gas under the influence of small white noise on the charge kinetics equations are investigated in detail. We may be confirmed in intention that considered problems allow more effectively to solve the tasks for planning and making of realistic practicable nuclegens in the future.

\section{References}

[1] Tertychny-Dauri, V.Yu. (1996) Hyperreactive Cosmodynamics and Physics of Compact Accelerators. Institut tochnoy mechaniki i optiki, Saint-Petersburg. (In Russian)

[2] Tertychny-Dauri, V.Yu. (2017) Solution of Nuclear Electrodynamics Equations Taking into Account $\beta$-Charged Eradiation. Open Access Library Journal, 4, e3794. https://doi.org/10.4236/oalib.1103794

[3] Tertychny-Dauri, V.Yu. (2018) Nuclear Electromagnetic Generator: Mathematical Model on Toroidal Vacuum Scheme. Open Access Library Journal, 5, e4524. https://doi.org/10.4236/oalib.1104524

[4] Harrer, J.M. (1963) Nuclear Reactor Control Engineering. D. Van Nostrand Company, Inc., Princeton, Toronto, London, New York.

[5] Keepin, G.R. (1965) Physics of Nuclear Kinetics. Addison-Wesley Publishing Company, London.

[6] Dementyev, B.A. (1973) Kinetics and Regulation of Nuclear Reactors. Atomizdat, Moscow. (In Russian)

[7] Petrov, B.N., Ed. (1974) Dynamics and Control of Nuclear Rocket Engine. Atomizdat, Moscow. (In Russian)

[8] Tertychny-Dauri, V.Yu. (1993) Adaptive Integrodifferential Algorithms of Stochastic Optimization for Dynamical Systems. Differential Equations, 29, 4, 610-616. (In Russian)

[9] Tertychny-Dauri, V.Yu. (2008) Galamech, Vol. 2, Stochastic Mechanics. Fizmatlit, Moscow. (In Russian)

[10] Aizerman, M.A. (1974) Classical Mechanics. Nauka, Moscow. (In Russian)

[11] Wentzel, A.D. and Freidlin, M.I. (1979) Fluctuations in Dynamical Systems under the Action of Small Random Perturbations. Nauka, Moscow. (In Russian)

[12] Feynman, R.P. and Hibbs, A.R. (1965) Quantum Mechanics and Path Integrals. McGraw-Hill Companies, Inc., New York. 